\title{
Milliliter per Cage per Day
}

National Cancer Institute

\section{Source}

National Cancer Institute. Milliliter per Cage per Day. NCI Thesaurus. Code C73751.

A dose calculation unit expressed in milliliter(s) per cage per period of time equal to twenty-four hours. 